\title{
SOBRE LA INTUICIÓN EN EL PENSAMIENTO DE KARL JASPERS
}

\author{
Cristóbal Holzapfel \\ Universidad de Chile \\ hcristob@yahoo.com
}

\begin{abstract}
RESUMEN / ABSTRACT
Examinamos los alcances de la intuición en el pensamiento de Karl Jaspers, para lo cual nos apoyamos especialmente en su Psicologia de las concepciones de mundo, de 1919. Lo que le preocupa allí al pensador es cómo se conforma una concepción de mundo (Weltanschaunng). Pues bien, ella se conforma, por el lado del sujeto, de actitud (Einstellung) y, por el lado del objeto, de imagen de mundo (Weltbild). Lo decisivo, en todo caso, es la actitud, ya que es ella la que se da a sí misma una imagen de mundo. Entre actitud del sujeto e imagen de mundo objetual hay las posibilidades de fisiónfusión-superación. A la actitud racional la caracteriza la fisión, ya que tiene una relación distante de oposición con el objeto, a la actitud mística la superación de la relación sujeto-objeto, y nuestra actitud intuitiva la fusión y una máxima cercanía con el objeto, en cierto modo, un hacerse uno con él. Al mismo tiempo, a la intuición la caracteriza la captación inmediata e instantánea de lo esencial de algo, y la acompaña el supuesto de la verdad que se devela con el descubrimiento, o más bien, la capacidad descubridora que también le sería propia.
\end{abstract}

Palabras Clave: intuición, concepción de mundo, sujeto-objeto, verdad.

\section{ON INTUITION IN THE THOUGHT OF KARL JASPERS}

We examine the scope of intuition in the thought of Karl Jaspers, for which we rely especially on his "Psychology of Worldviews", 1919. What worries the thinker there, is how a worldview (Weltanschaunng) conforms. Well, it conforms, on the side of the subject, with attitude (Einstellung) and, on the side of the object, image of the world (Weltbild). What is decisive, in any case, is the attitude, since it is she who gives herself an image of the world. Between the attitude of the subject and the image of the objetual world there are the possibilities of fission, fusion and overcoming. A rational attitude is characterized by fission, since it has a distant relationship of opposition to the object, while the mystical attitude the overcoming of the subject-object relationship.And our intuitive attitude is distinguished by fusion and a maximum closeness to the object, in a certain way, to become one with him. At the same time, intuition is characterized by the immediate and instantaneous grasp of the essential of something, and it is accompanied by the assumption of the truth that is revealed by the discovery, or rather by the discovering capacity that would also be its own.

KEYWORDS: Intuition, worldview, subject-object, truth. 
RA Cabe destacar que el pensamiento de Karl Jaspers -especialmente en su Psicología de las concepciones de mundo, Psychologie der Weltanschaunnen, 1919- tiene la particularidad de romper con la concepción puramente cognitiva con que ha solido plantearse la relación entre el sujeto y el objeto ${ }^{1}$.

Se podría decir con toda seguridad, sobre esta base, que la relación sujeto-objeto se refiere y abarca toda relación del enlace entre ser humano y mundo, trátese de una relación activa o contemplativa, entusiástica, mística, autorreflexiva, racional, estética o precisamente intuitiva.

La relación sujeto-objeto es eminentemente tensional. Podríamos decir que sujeto y objeto actúan al modo de polos, entre los cuales puede haber no solo fisión, sino también fusión. De hecho, como veremos, la intuición involucra más bien fusión, incluso entre todas las variaciones del vínculo entre hombre y mundo, la que más; en cambio, la racionalidad claramente implica más bien fisión. Pero, luego la mística o el entusiasmo suponen enfáticamente superación de la relación sujeto-objeto, aunque entre una y otra de distinta manera.

De este modo, la relación sujeto-objeto se presenta de acuerdo a la tríada fisiónfusión-superación. Advirtamos que, salvo la excepción e innovación que establece Jaspers en esta perspectiva, tradicionalmente se ha tenido en cuenta nada más que la fisión o escisión sujeto-objeto, ya que precisamente se ha circunscrito esta relación nada más que a un catastro cognitivo. Aun en Heidegger esto se mantiene, ya que en su pensamiento, en vistas de las insuficiencias que se declaran con la fisión sujeto-objeto, que a fin de cuentas es una relación de oposición con lo ente, con cosas o personas, el pensador de Friburgo considera solo la posibilidad de superación de aquella relación.

Si contrastamos el pensamiento de Karl Jaspers con el de Martin Heidegger, que fueran íntimos amigos especialmente entre 1920 y 1934, el de Heidegger, en lo que atañe a la relación sujeto-objeto, está marcado por una, a mi modo de ver, "obsesiva" tendencia a la superación de la relación sujeto-objeto. Su pensamiento del ser así lo exige. El pensamiento de Jaspers, en cambio, es más abierto a toda la extraordinaria riqueza que hay en esta relación.

Ahora bien, como nuestro tema de la intuición está principalmente abordado en la Psicología de las concepciones de mundo, lo que es precisamente una concepción de mundo, una cosmovisión, una Weltanschauung, consta de un componente subjetivo, entendido como actitud (Einstellung) y un componente objetivo entendido como imagen de mundo (Weltbild). Si al filósofo, anterior a Jaspers, Wilhelm Dilthey, le debemos un pensamiento de las concepciones de mundo ${ }^{2}$, con Jaspers éstas son miradas más bien

1 Jaspers, Karl, Psychologie der Weltanschauungen, München, Piper, 1985. En adelane 'PdW'. Las traducciones son mías. /Psicología de las concepciones del mundo, trad. de Mariano Marín. Madrid, Gredos: 1967.

2 Dilthey, Wilhelm, Weltanschaunngslehre (Teoría de las concepciones de mundo). Göttingen: Vandenhoeck\&Ruprecht, 1991. 
hacia dentro, atendiendo a sus componentes, a saber, precisamente un encuentro ente una actitud subjetiva y una imagen de mundo objetiva.

¿Dónde encontramos la actitud intuitiva en la obra de Jaspers sobre las Weltanschauungen? Pues en el contexto de las actitudes objetivas, las actitudes volcadas al objeto, las cuales pueden ser, entre otros: actitud activa que no deja-ser al objeto, puesto que está únicamente preocupada de su transformación, y actitud contemplativa que "deja ser" al objeto, pudiendo ser éstas las actitudes racional, estética y justamente intuitiva. En contraste con ello, la actitud activa, propia del técnico, del ingeniero, pero también del político, ve al objeto que se presente, en el caso del ingeniero el río sobre el que ha de construirse un puente, en el caso del político la comunidad, como algo para ser transformado y que ejerce resistencia. Esto quiere decir que la actitud activa no "deja ser" al objeto. Ahora bien, ambas actitudes, activa o contemplativa, son conformadoras de diversas concepciones de mundo. En el caso de la actitud activa, ella se da a sí misma una imagen de mundo sénsoro-espacial o mecánica, en cambio la actitud contemplativa puede darse a sí misma una imagen de mundo anímico-cultural.

Veamos cómo Jaspers caracteriza la intuición:

En la actitud intuitiva se ve, se aprehende, se vivencia el sentimiento gozoso de la plenitud y de lo sin-límite. No todo es reconocido como verdadero y rápidamente subsumido bajo categorías conocidas (con el sentimiento de no vivenciar nada nuevo, de no ver nada esencial, como, por ejemplo, lo experimenta la ceguera intuitiva en la movilidad racional); sino que, entregándose, se contempla, esperando, se aprehende, el ver es vivenciado como vivencia "creativa" del crecer. Resulta claro que la voluntad, la finalidad, el consciente trazado de metas, entorpece y estrecha, que el ser-dado es una habilidad y don de la propia naturaleza, mucho más que el mérito del trazado de metas de la voluntad, disciplina y principios, a no ser del principio de entregarse por de pronto sin cuestionamiento, cuando el instinto dice que algo debe ser intuitivamente puesto de manifiesto (PdW, p. 64-65).

Claramente lo que está en juego con la intuición ante todo es un entregarse a que algo se nos muestre ("entregándose, se contempla, esperando, se aprehende") y por lo tanto que eso suceda no depende de cierta voluntad o disciplina nuestra; la intuición, y junto con ello, la actitud intuitiva que podemos tener, es más bien una habilidad, en tanto don natural. De ahí también que lo que se revela en la intuición tampoco puede ser de inmediato subsumido en ciertas categorías, teorías o sistemas, como es lo propio de la actitud racional:

La actitud intuitiva no es un veloz mirar hacia algo, sino un sumergirse. No es corroborado nuevamente con una mirada lo que ya sabíamos, sino que nos apropiamos de algo nuevo, pleno en un proceso de una intuibilidad en desarrollo (PdW, p. 65).

Probablemente la asociación que arriba se establecía entre intuición y crecimiento ahora se aclara mejor, ya que la intuición tiene particular relación con lo nuevo, y agreguemos aquí, precisamente por su carácter de ser descubridora, que le es afín. Efectivamente, 
si la intuición no subsume lo que ve bajo categorías ya conocidas, ni puede hacerlo, en ella hay crecimiento, porque hay descubrimiento.

Más que todo lo dicho recién, corresponde destacar que en verdad el conocimiento, el saber (todo conocimiento y todo saber) avanza exclusivamente por la intuición. Solo ella tiene esa afinidad con lo nuevo, y al mismo tiempo una virtud descubridora.

Pero, hay más todavía en la última cita. Jaspers nos ayuda a corregir el error inveterado de la asociación de la intuición con su supuesto carácter veloz. Es cierto que el primer momento de la intuición semeja la virtud propia de la fotografía de captar velozmente lo característico, lo esencial de algo en un instante (abertura y cierre del obturador) (y por eso también la expresión “instantánea"). Pero, lo que sigue a ese primer instante es un sumergirnos en lo intuido, y ello diríamos al modo de un instante que se eterniza. Justamente el instante, como el eterno presente del ser parmenídeo, no tiene por qué ser atómico.

Recordemos que una de las citas anteriores comenzaba diciendo: "En la actitud intuitiva se ve, se aprehende, se vivencia el sentimiento gozoso de la plenitud y de lo sin-limite". Jaspers plantea que cada cosa, cada fenómeno particular es infinito; exceptúa de ello solo a los artefactos y productos humanos. Mas, si se trata, por ejemplo, de una naranja, ella es infinita, diríamos "hacia dentro". Es decir, en la exploración de cada cosa o persona, y aunque sea una gota de agua, nunca encontraríamos un límite, un término. A partir de ello se explican a la vez algunos caracteres relevados más arriba, como, por ejemplo, que la intuición nos lleve a sumergirnos en su objeto, que ese instante se eternice, que no acabemos nunca de seguir descubriendo lo que hay en su interior.

La mencionada instantaneidad supone a la vez la pareja inmediatez de la intuición, en contraste con la mediación propia de la razón. Lo que intuimos es precisamente de una vez. Solemos tener la intuición de que supimos en un instante algo determinado, como al decir que de una vez "se nos aclaró la película", que "captamos que este lugar no es para nosotros", y demás. O en el caso de la fotografía, se capte de un solo golpe el instante en el que se retrata todo un conjunto complejo de cosas, tal vez unos niños que corren cerro abajo en alguno de los cerros de Valparaíso (como en la fotografía de Sergio Larraín), el rostro de una niña afgana en un campo de refugiados en Afganistán, bajo las consecuencias de la desolación, como es la foto de la niña Sharbat Gula, realizada por Steve Curry en 1984. Giorgio Agamben reflexiona en Profanaciones sobre esta característica de la fotografía, tomando en cuenta la que históricamente es la primera de todas, la de Monsieur Daguerre ${ }^{3}$.

Por lo tanto, hay que tener cuidado con el tema de la inmediatez y del parejo instante, ya que no necesariamente se limita esto a algo temporal, mensurable cuantitativamente, como podría presumirse. Si fuera así, la inmediatez de la intuición y su aliado instante

Agamben, Giorgio, Profanierungen, Frankfurt a/M: Suhrkamp, 2005. p. 8. / Ed.cast.: Profanaciones, trad. de Flavia Costa y Edgardo Castro. Buenos Aires: Adriana Hidalgo, 2005, "El día del juicio", p. 29. 
corresponderían a la mínima unidad, a saber, en centésimas o incluso milésimas de segundo. No, de ninguna manera se trata aquí de esa unidad mínima, por lo demás, inalcanzable para nosotros, justamente porque el tiempo es infinitamente divisible, sino de un instante en el que nos sumergimos, dándonos tiempo para ello. Es por ello que lo que estamos diciendo se puede comparar más bien con esas fotos con tiempo, en las que, por ejemplo, las luces de la ciudad se ven como líneas o rayos.

A continuación, y en apretada síntesis, Jaspers nos presenta la tríada, que nosotros hemos caracterizado como de fisión-fusión-superación y su relación respectiva con la actitud racional, intuitiva y mística:

Si bien todo lo intuitivo se basa en la fisión sujeto-objeto, sin embargo, el movimiento va entre sujeto y objeto de un modo tal que hay una conciencia de la cercanía, de la co-pertenencia, del parentesco con el objeto, mientras que la actitud racional produce la gran distancia y la actitud mística levanta completamente la fisión sujeto-objeto (PdW, p. 65).

Sopesando lo anterior, podemos reconocer que la intuición, al captar lo esencial de algo, a veces de una situación, o incluso de un conjunto altamente complejo, en verdad ello es posible en el ámbito de una cercanía con el objeto, a diferencia de la actitud racional que, con su afán de explicación y de dar razones de qué es y por qué es lo que está enfrente, aleja el objeto.

Reparemos en la relevancia ontológica que se revela en esto del espacio, y para decirlo más precisamente con el Heidegger de Ser y tiempo, de la espacialidad, esto es, aludiendo al modo como existencialmente espacializamos el espacio de distinta forma. Relacionando lo planteado por Jaspers respecto de las actitudes racional e intuitiva, podríamos decir (con apoyo en Heidegger) que la primera actitud mantiene al objeto en una lejanía, en cambio la actitud intuitiva lo "desaleja", manteniéndolo en una cercanía ${ }^{4}$.

Por otra parte, Jaspers considera que suele reducirse la intuición a lo meramente sensorial. Mas, aquí cabría agregar que ello tiene que ver con el término alemán para 'intuición', que es 'Anschaunng', que en efecto se puede aplicar predominantemente a lo que experimentamos con los sentidos. Nuestro término español 'intuición', sin embargo, no lo usamos así casi nunca. Para nosotros la intuición ya de por sí es de carácter intelectual. Y este punto es el que está justamente en juego aquí: que habría no solo una intuición sensorial, sino intelectual o espiritual.

De hecho, la intuición intelectual (intellektuelle Anschauung) corresponde nada menos que a uno de los puntos capitales que se barajan en la constitución del idealismo alemán, ya que con Hegel y su Differenzschrift (Escrito de la diferencia) de cara a la

$4 \quad$ Heidegger, Martin, Sein und Zeit, Tübingen: Niemeyer, 1977; en lo sucesivo 'SuZ'. / Ed. cast.: Ser y tiempo, trad. de Jorge E. Rivera, Santiago: Universitaria, 1997; en lo sucesivo 'Syt-R'. Tb. Ser y tiempo. Madrid: Trotta, 2003, parágrafos 22, 23, 24. 
incognoscibilidad de la cosa en-sí de Kant, habría la posibilidad de una intuición no sensorial, sino estrictamente intelectual, que sería capaz de captarla 5 .

Y luego, agrega nuestro pensador otro punto importante: que aquello que se nos muestra en la intuición es incomunicable. Ciertamente podemos intentar develar qué es lo que intuimos de algo cuando lo intuimos, pero en última instancia ello es incomunicable. Y, nótese que ello vale tanto para los colores que vemos como para objetos más complejos. En esto naturalmente se diferencia la intuición de lo racional.

De todos modos, hay ciertos recursos técnicos para superar esa incomunicabilidad, pero son siempre limitados. En el caso de la intuición sensorial (y también de las intuiciones internas) puede haber reproducciones, dibujos, pinturas, sonidos producidos por instrumentos musicales, y ciertamente palabras.

Respecto de esta captación de lo esencial, propia de la intuición, se revela a la par cierto vínculo que podríamos llamar "natural" que hay en el hombre con relación a lo verdadero, ya que indudablemente lo que se manifiesta en la intuición al mismo tiempo se supone que es ni más ni menos que la verdad de algo. Ahora bien y por eso mismo, hay que anotar que desde luego todo lo que involucra la intuición y sus extraordinarias aptitudes da pie a que ella se transforme en algo digno de emular y entonces todo el mundo pretende poseer esta virtud, lo que naturalmente se presta para el abuso. Es un lugar común, por ejemplo, la intuición femenina, y si ella tiene alguna base, sucede que toda mujer que se precie de tal, suele pretender que por el solo hecho de ser mujer ya posee este don. Y así se podrían citar numerosos otros ejemplos de índole similar.

Recapitulemos lo analizado de la intuición:

1. Lo que descubrimos mediante la intuición es, en general, algo nuevo, y por eso no subsumible de inmediato bajo categorías racionales conocidas.

2. Si bien, la intuición es inmediata e instantánea, esto hay que entenderlo al modo de un sumergirse en el objeto, perpetuando el instante.

3. Ello se cumple, máxime desde el momento que lo que intuimos es incomunicable. Desde luego que podemos dar explicaciones de eso intuido, pero éstas no hacen sino rodearlo.

4. A la actitud intuitiva la caracteriza a su vez una receptividad, ya que en ella estamos a la espera y entregados a que algo se nos revele.

5. También es propio de ella una cercanía con el objeto, en contraste con la actitud racional, que más bien impone una distancia respecto del objeto.

6. Ahora bien, tengamos también en cuenta que hay intuición sensorial como espiritual o intelectual, mas Jaspers agrega a ello que aun elevándonos a

Hegel, Diferencia entre el sistema de filosofía de Fichte y el de Schelling. Madrid: Alianza, 1989. 
lo espiritual, lo sensorial no desaparece; en otras palabras, lo sensorial se mantiene en las intuiciones espirituales de lo más sutil, como las ideas.

7. Pero, cabe hacer notar que, dado que entre intuición y verdad hay, o, más bien, se supone que hay un nexo con la verdad, en otras palabras, que lo que intuimos lo suponemos verdadero, entonces aquel que cree tener esto supone que lo posee al modo de un don. Un ejemplo extremo de ello sería lo que atañe al don de la profecía o de la clarividencia. Supuestamente lo que estas personas dotadas ven es, justamente al modo de una intuición, de un golpe de vista.

Independientemente de estos casos extremos, que, al parecer son efectivos y auténticos, la intuición permite aprehender cosas, fenómenos, situaciones y asuntos muy complejos de una vez, de un solo golpe, como si un rayo de luz nos atravesara, y por lo mismo ello se presta para ciertas presunciones y abusos.

Mas, con prescindencia de estos "abusos" interesa al menos reconocer que hay la intuición y que efectivamente, como hablamos aquí de una "actitud intuitiva", esta actitud puede ser más fuerte y claramente reconocible en unas que en otras personas. Y sin duda de mayor relevancia es que esa captación de lo esencial, propia de la intuición, requiere de una pasividad, de un estar entregado a que algo se me revele, pero agreguemos, una entrega tal sin esperar algo en concreto, ya que entonces se daría el peligro de que con nuestra expectativa e ilusión construyéramos algo que supuestamente se nos está revelando.

Visto de esta forma, de lo que se trata, más que de una receptividad, es lisa y llanamente de una pasividad, ya que es ella la que mejor expresa una detención de la actividad, por sobre todo racional, y agreguemos también, emocional, del sujeto, lo cual permite que lo otro, el fenómeno, se le revele en lo esencial a la intuición.

Importa también atender muy especialmente a que esa captación intuitiva se dé de una vez, siendo esto algo que la razón no puede, ya que ésta va paso a paso intentando conquistar su objeto.

Ahora bien, si es propia de la intuición la receptividad y la pasividad, y que ellas permiten que el fenómeno, que sea el caso, una persona, una cosa, una situación, un evento, un acontecimiento, se muestre por sí mismo, evitando a la vez que se entrometa aquí la actitud racional, que con apoyo en la sola razón o la experiencia, arroja cierta red conceptual, dejando atrapado en alguno de sus retículos al fenómeno, entonces lo que se baraja con la intuición tiene todas las marcas propias del método fenomenológico inaugurado por Edmund Husserl.

Tal vez ello explica que Jaspers sea considerado también como fenomenólogo, aunque él mismo no se haya reconocido como tal y haya estimado tener más bien una relación indirecta con la fenomenología. 
Referencias bibliográficas

Agamben, Giorgio (2005), Profanierungen. Frankfurt a/M: Suhrkamp, 2005 / Ed.cast.: Profanaciones, trad. de Flavia Costa y Edgardo Castro. Buenos Aires: Adriana Hidalgo.

Dilthey, Wilhelm (1991), Weltanschauungslehre (Teoria de las concepciones de mundo).Göttingen: Vandenhoeck\&Ruprecht.

Hegel (1989), Diferencia entre el sistema de filosofía de Fichte y el de Schelling. Madrid: Alianza.

Heidegger, Martin (2003), Sein und Zeit, Tübingen: Niemeyer, 1977; en lo sucesivo 'SuZ'. / Ed. cast.: Ser y tiempo, trad. de Jorge E. Rivera, Santiago: Universitaria, 1997; en lo sucesivo 'Syt-R'. Tb. Ser y tiempo. Madrid: Trotta.

Jaspers, Karl (1967), Psychologie der Weltanschauungen (PdW), München, Piper, 1985. En adelane 'PdW'. Las traducciones son mías. /Psicología de las concepciones del mundo, trad. de Mariano Marín. Madrid: Gredos. 